superior to established prognostic indicators, and might be more useful than left-ventricular ejection fraction in assessing patients' risk.

Original article Doust JA et al. (2005) How well does B-type natriuretic peptide predict death and cardiac events in patients with heart failure: systematic review. $B M J$ [doi: 10.1136/bmj.330.7492.625]

\section{Leukocyte count in cardiovascular risk assessment}

Inflammation has been implicated in the pathogenesis of atherosclerosis and so might provide useful prognostic information. Recent work by Margolis and colleagues attempted to define the relationship between white blood cell (WBC) count and cardiovascular events and mortality in postmenopausal women.

The multicenter study, part of the Women's Health Initiative Observational Study, included 72,242 women aged 50-79 years, all of whom underwent WBC count assessment at baseline. Subsequent cardiovascular events (death from coronary heart disease, nonfatal myocardial infarction, and stroke) and total mortality were then monitored during a mean follow-up of 6.1 years.

After adjustment for variables such as smoking and hypertension, women with WBC counts in the fourth quartile $\left(6.7-15.0 \times 10^{9}\right.$ cells $\left./ \mathrm{l}\right)$ were at more than double the risk of cardiovascular death compared with those in the first quartile $\left(2.5-4.7 \times 10^{9} \mathrm{cells} / \mathrm{I}\right)$. These women were also at a significantly higher risk for total mortality, stroke, and nonfatal myocardial infarction. Even after further adjustment for Creactive protein and lipids, the WBC count was significantly associated with coronary events.

Concluding that WBC count appears to be an independent predictor of cardiovascular events and total mortality in older women, Margolis and colleagues propose that a threshold value of $6.7 \times 10^{9}$ cells/l is appropriate as an indicator of elevated risk. They suggest that this widely available and convenient marker might help to identify high-risk individuals who are not defined as such by current methods.

Original article Margolis KL et al. (2005) Leukocyte count as a predictor of cardiovascular events and mortality in postmenopausal women. Arch Intern Med 165: 500-508

\section{Value of screening for abdominal aortic aneurysms}

A recent Danish study has demonstrated that screening for abdominal aortic aneurysms in older men saved one life per 352 men screened, reducing specific mortality by $67 \%$.

Lindholt et al. randomized men aged 65 years and over to screening by abdominal ultrasonography $(n=6,333)$ or no screening $(n=6,306)$. Mortality due to abdominal aortic aneurysm, total mortality, number of ruptured aneurysms, and details of operations were then recorded in both groups, over a mean follow-up of 52 months.

The screening attendance rate was $76.6 \%$, and $4.0 \%$ of those screened (191 men) had abdominal aortic aneurysms. In an intention-to-screen analysis, abdominal aortic aneurysm was recorded in 9 men in the screening group, compared with 27 in the control group (hazard ratio $0.33,95 \% \mathrm{Cl}$ 0.16-0.71). In addition, all-cause mortality and death from causes other than abdominal aortic aneurysm were each $8 \%$ lower in the screening group. Total mortality among those who were randomized to screening but did not attend was almost twice the level recorded among those who did attend. Participants in the screening group underwent over four times more elective operations and four times fewer emergency operations than those in the control group.

Noting that these results are consistent with other studies in the UK and Western Australia, Lindholt et al. conclude that mass screening for abdominal aortic aneurysm appears to reduce mortality and that studies into its cost-effectiveness are warranted.

Original article Lindholt JS et al. (2005) Screening for abdominal aortic aneurysms: single centre randomised controlled trial. $B M J$ [doi: 10.1136/bmj.38369.620162.82]

\section{Do thiazide diuretics increase the risk of gallbladder disease?}

Following reports linking thiazide diuretics with increased biliary cholesterol saturation, it has been postulated that these drugs increase the risk of gallstone development. Leitzmann and colleagues have investigated this in their recent study in the US.

A total of 81,351 women aged $30-55$ years were enrolled in this 20-year study. Use of 